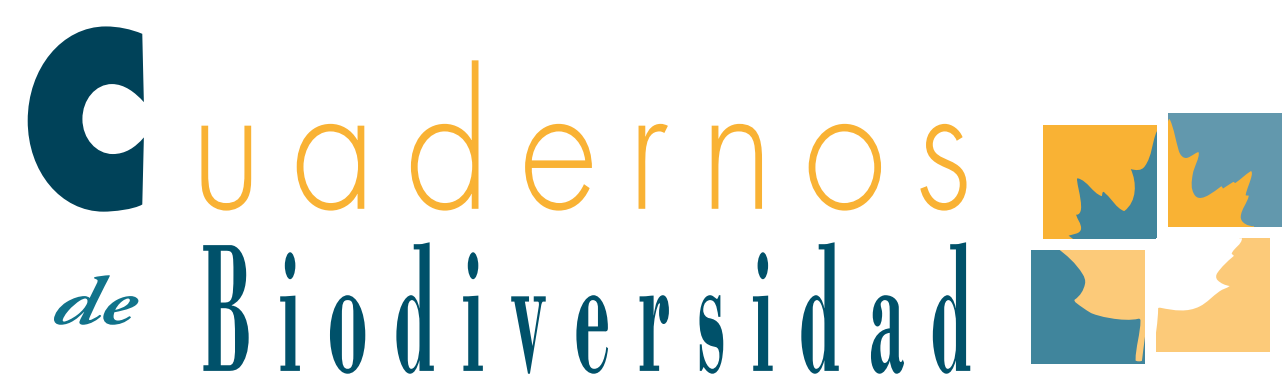

Cuadernos de Biodiversidad 58 (2020): 9-22

I.S.S.N.: 2254-6I $2 \mathrm{X}$

DOI:IO.I4I98/CDBIO.2020.58.02

\title{
Programa de conservación y recuperación de Boerhavia repens L. (Nyctaginaceae), especie en peligro de extinción en la Comunidad Valenciana (España)
}

Conservation and recovery program for Boerhavia repens L. (Nyctaginaceae), an endangered species in the Valencian Community (Spain)

Inmaculada Ferrando-Pardo, P. Pablo Ferrer-Gallego, Francisco J. Albert i, Joan PérezBotella, Roger Carchano, Carlos Gisbert \& Emilio Laguna

Autor:

I. Ferrando-Pardoo ${ }^{\mathrm{I}, 2 *}$, P.

P. Ferrer-Gallego ${ }^{1,2}$, F. J.

Albert $^{1,2}$, J. Pérez-Botella ${ }^{2,3}$, R. Carchano ${ }^{2,3}$, C. Gisbert ${ }^{2,3}$ \& E. LAGUNA ${ }^{\mathrm{I}}$

I Generalitat Valenciana, Servicio de Vida Silvestre, Centro para la Investigación y la Experimentación Forestal (CIEF). Valencia, España.

2 Generalitat Valenciana, VAERSA. Valencia,España.

3 Generalitat Valenciana, Servicio de Vida Silvestre, SSTT Alicante. Alicante, España.

*endemica_cief@gva.es

Recibido: 15/05/2019

Aceptado: 15/06/2020

Publicado: 15/07/2020

LICENCIA:

Este trabajo se publica bajo una Licencia Creative Commons

Reconocimiento 4.0 Internacional.

\section{(c) (i)}

Cómo citar:

Ferrando-Pardo, I., Ferrer-Gallego, P.P., Albert, F.J., Pérez-Botella, J., Carchano, R., Gisbert, C., Laguna, E.. (2020). Programa de conservación y recuperación de Boerhavia repens L.(Nyctaginaceae), especie en peligro de extinción en la Comunidad Valenciana (España). Cuadernos de Biodiversidad (58), 9-22. https://doi. org/10.14198/cdbio.2020.58.02

\section{RESUMEN}

Boerhavia repens está incluida en el Catálogo Valenciano de Especies de Flora Amenazadas de la Comunidad Valenciana (Orden 6/2013) en la categoría “En peligro de Extinción”. La única población española conocida está localizada en Teulada (Alicante, España), siendo la segunda población conocida en toda Europa. La población alicantina desapareció en 2012, y hasta el momento se han realizado 10 plantaciones en la provincia de Alicante. De un total de 615 plantas introducidas se ha registrado una tasa de supervivencia del 8,7\% (54 plantas) en el último censo. Diferentes trabajos con la germinación de las semillas y cultivo de esta planta han sido realizados en el CIEF.

Palabras clave: Alicante; especie en peligro de extinción; conservación ex situ e in situ; semillas; translocaciones.

\section{ABSTRACT}

Boerhavia repens is included in the Valencian Catalogue of Threatened Species of the Valencian Community (Order 6/2013) in the category "In danger of extinction". The only known Spanish population is located in Teulada (Alicante, Spain), being the second known population in Europe. This population disappeared in 2012, and so far 10 plantations have been made in the province of Alicante. A total amount of 615 plants were translocated and a survival rate of $8,7 \%$ (54 plants) was observed in the latest census. Different works with the germination of seeds and cultivation of plants have been carried out at CIEF.

Key words: Alicante province; endangered species; ex situ and in situ conservation; seeds; translocations. 


\section{INTRODUCCIÓN}

Boerhavia repens L. es una especie perteneciente a la familia Nyctaginaceae con representación en la flora española y valenciana tan sólo a través de una única población conocida, situada en el cuadrante nororiental de la provincia de Alicante (Crespo 2004, Bolòs et al. 2005, Mateo \& Crespo, 2014). Esta especie se distribuye por los continentes africano y asiático (Maire 1962, Codd 1966, Chen \& Wu 2007, AFRICAN PLANT DATABASE 2012), y también aparece en Europa, pero de manera muy puntual, donde solo se conoce de Sicilia (al parecer como adventicia) y en la mencionada localidad alicantina, localizada concretamente en la "Serra de les Cel-letes", en el término alicantino de Teulada (Soler 1995).

Boerhavia repens es una especie de porte subarbustivo con numerosos tallos delgados y algo leñosos, muy ramificados, que crecen tendidos en el suelo, algunos de ellos erectos, llegando a medir más de 1 $\mathrm{m}$ de longitud; las hojas son opuestas y pecioladas, de tamaño variable; las flores, reunidas en inflorescencias paniculadas, son muy pequeñas $(2-5 \mathrm{~mm}$ de diámetro) y de color rosado; el fruto (epicarpio) es seco, indehiscente, monospermo y de color marrón rojizo cuando está maduro, recubriéndose de una sustancia mucilaginosa cuando aumenta la humedad ambiental o entra en contacto con el agua (Maire 1962, Struwig \& Siebert 2013). Desde el punto de vista taxonómico, se han descrito varios táxones infraespecíficos, estando en la actualidad dos subespecies reconocidas: subsp. repens y subsp. diandra (L.) Maire \& Wieller. La última distribuida por Egipto, África tropical, Palestina y Pakistán (Maire 1962, Klopper et al. 2006, Struwig \& Siebert 2013). Las plantas valencianas han sido identificadas como pertenecientes a la subespecie tipo (Soler 1995, Crespo 2004).

En la población valenciana, las plantas pierden la parte aérea en la época de mayor estrés, que es el invierno, pero conserva la parte subterránea (cepa leñosa) viva. La floración comienza en junio y se prolonga hasta octubre, siendo la fructificación desde junio hasta octubre. La mayoría de especies del género Boerhavia no parecen tener preferencias edáficas definidas, pero $B$. repens subsp. repens se ha relacionado con altos niveles de Magnesio (Struwig $\&$ Siebert 2013). En el territorio valenciano habita en repisas de roquedos calco-dolomíticos, algo alterados, en zonas cálidas muy expuestas a la radiación solar, dentro del dominio de los carrascales termomediterráneos (código Natura 2000: 8210 "Pendientes rocosas calcícolas con vegetación casmofítica”). Con frecuencia, las poblaciones asiáticas y algunas africanas aparecen en zonas arenosas y pedregosas, sobre suelos con buen drenaje (Struwig \& Siebert 2013).

Por otro lado, comentar que esta especie ha sido empleada tradicionalmente con fines medicinales y alimentarios en la India y África, debido a sus múltiples propiedades derivadas de los glucósidos flavonoides y el alcaloide purnarnavina. Sus raíces se usan como diurético, cardiotónico, hepatoprotector, laxante, antihelmíntico, expectorante y antipirético. En África la bebida resultante de la decocción de raíces y hojas se utiliza también para tratar el asma, calmar las convulsiones, dolores estomacales, o como infusión abortiva. Las raíces hervidas y ralladas se aplican externamente para curar úlceras y llagas, y la planta molida se usa también externamente para los edemas. La savia de las raíces se aplica por vía ocular para tratar la infección parasitaria de la "elefantiasis". Entre los usos alimenticios, en Senegal y Mali las semillas se ańaden a los cereales y otras comidas. En África occidental las hojas se comen como verdura, preparadas en salsa, y en Nigeria las raíces se usan en repostería. Por otra parte, también se aprovechan los tallos de esta planta para dar de comer al ganado. Parte de esta información puede ser consultada en los trabajos publicados por Muzila (2006), Saikia et al. (2006), De Albuquerque et al. (2007) y Lehman et al. (2007).

En el territorio valenciano, en el momento de su descubrimiento, en la década de los años 90 del pasado siglo, se censó la población en una treintena de individuos, algunos de ellos jóvenes, en los que se observó una elevada producción de semilla. Los trabajos de seguimiento para el Proyecto AFA (Atlas y Libro Rojo de la Flora Vascular Amenazada), realizados a comienzos del presente siglo, incrementaron 
este valor hasta un total de 51 ejemplares, adultos en su mayoría. Sin embargo, los últimos censos realizados detectaron una fuerte reducción poblacional, no encontrándose ejemplares silvestres desde 2012. Así, desde el punto de vista de su estado de conservación a nivel nacional fue evaluada como especie "En Peligro Crítico" en el Atlas y Libro Rojo de la Flora Vascular Amenazada de España según los criterios UICN (2001) B1 ab(iii) + 2ab(iii); D (Crespo 2004). A nivel autonómico se encuentra incluida en el Catálogo Valenciano de Especies de Flora Amenazadas, en la categoría "En peligro de extinción” (Anónimo 2009, 2013, Aguilella et al. 2010).

Aunque las principales amenazas que se venían considerando para esta especie en la Comunidad Valenciana han sido el reducido número de ejemplares y escaso reclutamiento por una parte, y la posible actividad minera de las cercanas canteras de piedra (Crespo 2004), en la actualidad creemos que la desaparición de las plantas que constituían la población de Teulada ha sido posiblemente agravada por las escasas precipitaciones, o una conjunción de diferentes factores (véase lo comentado más abajo).

Ante este escenario ha sido necesario el diseño de un programa de conservación y recuperación que integrara diferentes medidas urgentes para el mantenimiento de este exclusivo material genético presente en el territorio valenciano, tanto in situ como ex situ. Aunque la inclusión de este tipo de poblaciones y especies en un listado de flora protegida pueda ser objeto de crítica, la falta de información filogeográfica recomienda aplicar el criterio de máxima precaución, y en consecuencia su inclusión en la herramienta normativa legal, algo que permite la conservación genética de una especie vegetal de gran rareza para la flora europea, independientemente del origen (autóctono vs. alóctono) de la población alicantina, algo que esperamos sea revelado en futuros estudios.

En el presente artículo se evalúa la situación actual de la especie y se exponen las acciones de conservación y recuperación llevadas a cabo por el equipo del Servicio de Vida Silvestre de la Generalitat Valenciana. Los trabajos in situ se han centrado en el diagnóstico y situación real de la especie en el hábitat natural (detección de amenazas, censos, georreferenciación, búsqueda de nuevos ejemplares, etc.) y acciones de translocaciones para la conservación (plantaciones). Las actividades ex situ están encaminadas a la conservación y análisis de las semillas, multiplicación y producción de ejemplares mediante su cultivo, y creación de colecciones de planta viva para abastecimiento de germoplasma.

\section{MATERIAL Y MÉTODOS}

\section{Conservación in situ. Censos y translocaciones de conservación}

Los censos realizados a lo largo del tiempo en la única población conocida así como a las poblaciones translocadas se han realizado mediante conteo directo de ejemplares.

Se han creado varias neopoblaciones en la Comunidad Valencia (Figura 5), a través de la introducción de ejemplares en el medio natural producidos en el vivero del Centro para la Investigación y la Experimentación Forestal de la Generalitat Valenciana (CIEF). Para la selección de los enclaves donde se realizaron las translocaciones se realizó una búsqueda de hábitats con características similares al hábitat natural conocida para la especie en la Comunidad Valenciana y que al mismo tiempo fueran monte de utilidad pública o territorios protegidos por la red de Microrreservas de flora de la Comunidad Valenciana. Algunas de las translocaciones que se han realizado pueden encuadrarse en reforzamientos de proximidad y neopoblaciones de seguridad, según lo expuesto por Laguna \& Ferrer-Gallego (2012).

\section{Conservación ex situ. Conservación de semi- llas y producción de planta en vivero.}

Los métodos empleados en la recolección, caracterización, conservación y germinación de las semillas, así como para el cultivo de plantas fueron los publicados por Ferrando-Pardo et al. (2008, 2016) y Ferrer-Gallego et al. (2013). El tipo de contenedor empleado fue bandeja de cultivo forestal Herku Ref. 
QP24T16 de 24 alveolos y 320 cc. de capacidad. El sustrato utilizado estaba compuesto por turba: fibra de coco: vermiculita, en proporciones 3:2:1. Transcurridos 6 meses desde la germinación y repicado a contenedores de cultivo, las plantas alcanzan un estado de desarrollo adecuado para ser plantadas en campo.

La producción de plantas de Boerhavia repens se ha beneficiado del soporte financiero del Fondo Europeo Agrícola del Fondo Rural (FEADER) en el marco de la Operación 8.5.3 "Conservación y desarrollo de la Red Natura 2000" como parte del Programa de Desarrollo Rural de la Comunitat Valenciana 2014 -2020.

\section{RESULTADOS Y DISCUSIÓN}

\section{Acciones de conservación in situ.}

\subsection{Monitoreo de la población natural.}

En la figura 1 se muestran los datos de los censos que se han registrado desde 1994, año en que fue descubierta esta especie con un total de 30 ejemplares. Desde esta fecha hasta 2002 se observa un incremento de ejemplares, de hasta 51 individuos, pero algunas de estas plantas eran muy jóvenes en el momento del censo, y podrían corresponder a ejemplares de Commicarpus africanus (Lour.) Dandy (Crespo, com. pers.), especie con la que convive y con la que es fácil de confundir en estado de plántula. Los censos de los siguientes años muestran un fuerte declive poblacional, con registros que no superan los 13 individuos a partir del año 2005. Desde 2012 no se han vuelto a encontrar ejemplares vivos silvestres. El último registro de esta población fue de 4 individuos (2012) no ocupando más de $2 \mathrm{~m} 2$. Los años 2015, 2016, 2017 y 2018 no han localizado ejemplares (véase Figuras 1, 2 y 3 ).

Los factores y amenazas que podrían haber marcado el rápido declive y finalmente la pérdida de la única población europea de Boerhavia repens podrían ser los siguientes:
- Escaso número de individuos de la población y plantas muy envejecidas. No se tiene constancia de reclutamientos anuales. Solo en 2 ocasiones, de un total de 13 datos censales, se han contabilizado nuevos ejemplares reclutados pero con bajos incrementos poblacionales, uno fue de 21 ejemplares nuevos y otro de 2 ejemplares con respecto al año anterior del censo.

- Actividad minera cercana dedicada a la extracción de gravas, arenas, arcilla y caolín (ver Figura 3). El polvo generado por la actividad minera se deposita en las superficies foliares de las plantas del entorno. No es descartable que afecte al desarrollo de las plántulas, impidiendo el reclutamiento de ejemplares. Tampoco es descartable que estas actividades podrían haber reducido enclaves potenciales para la expansión y colonización de esta especie dada la proximidad existente con la población (Crespo 2004).

- Sequía. Los periodos de sequía prolongados podrían haber reducido el tamaño de la población hasta su total desaparición.

\subsection{Translocaciones de conservación.}

Desde 2011 se han realizado 10 plantaciones mediante translocaciones de conservación para las que se han utilizado un total de 615 plantas producidas en los viveros del CIEF. Estas actuaciones han consistido en un refuerzo poblacional (MRF Serra de Cel-letes) y 9 introducciones (ver Tabla 1 y Figuras 4 y 5).

De las 10 translocaciones, 4 de ellas no han tenido resultados positivos y no ha habido supervivencia de los ejemplares introducidos en campo, en algunos casos ha sido debido a la predación de las plantas por conejos. El porcentaje de supervivencia medio de los ejemplares observados en las 10 translocaciones es de 11,8 $\pm 11,9$ trascurridos entre 1 y 3 años desde la plantación. La translocación con mayor supervivencia detectada al cabo de un año es la realizada en Bardalet (Elx) con 37,5\% (9 ejemplares) (Figuras 4 y 5). 


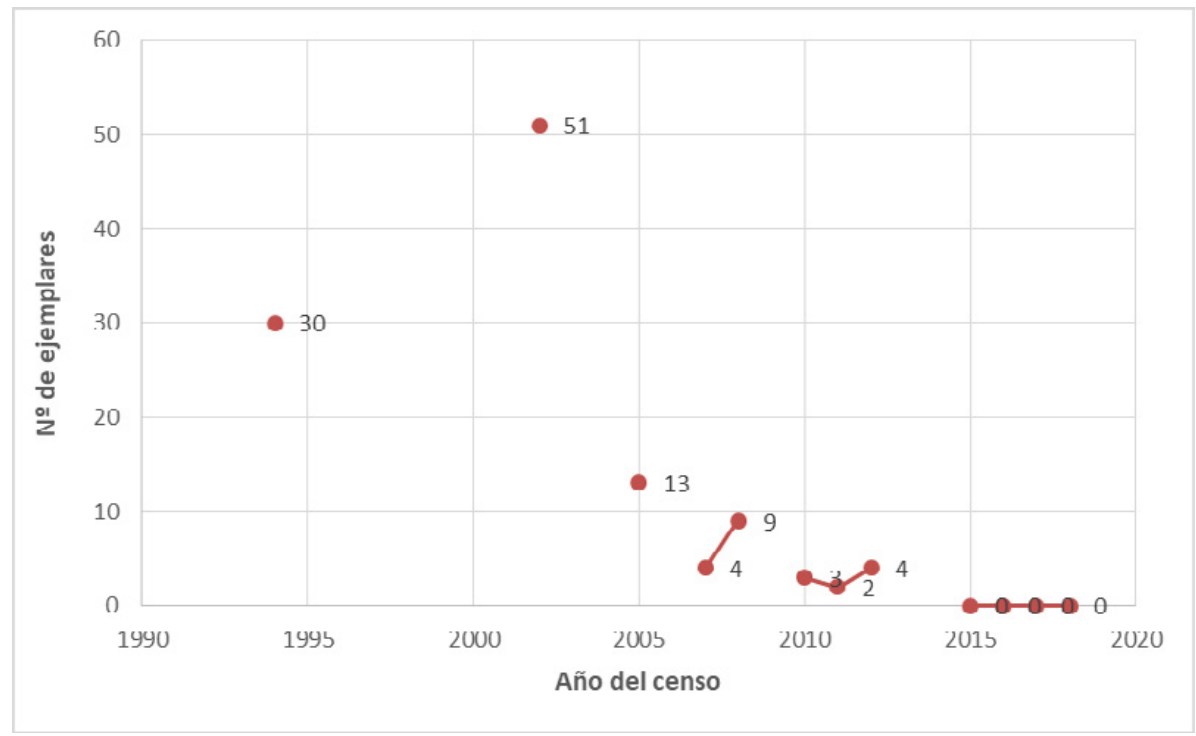

Figura 1. Evolución del número de ejemplares de la única población natural de Boerhavia repens en España, presente en Teulada (Alicante) desde 1994 hasta 2019.
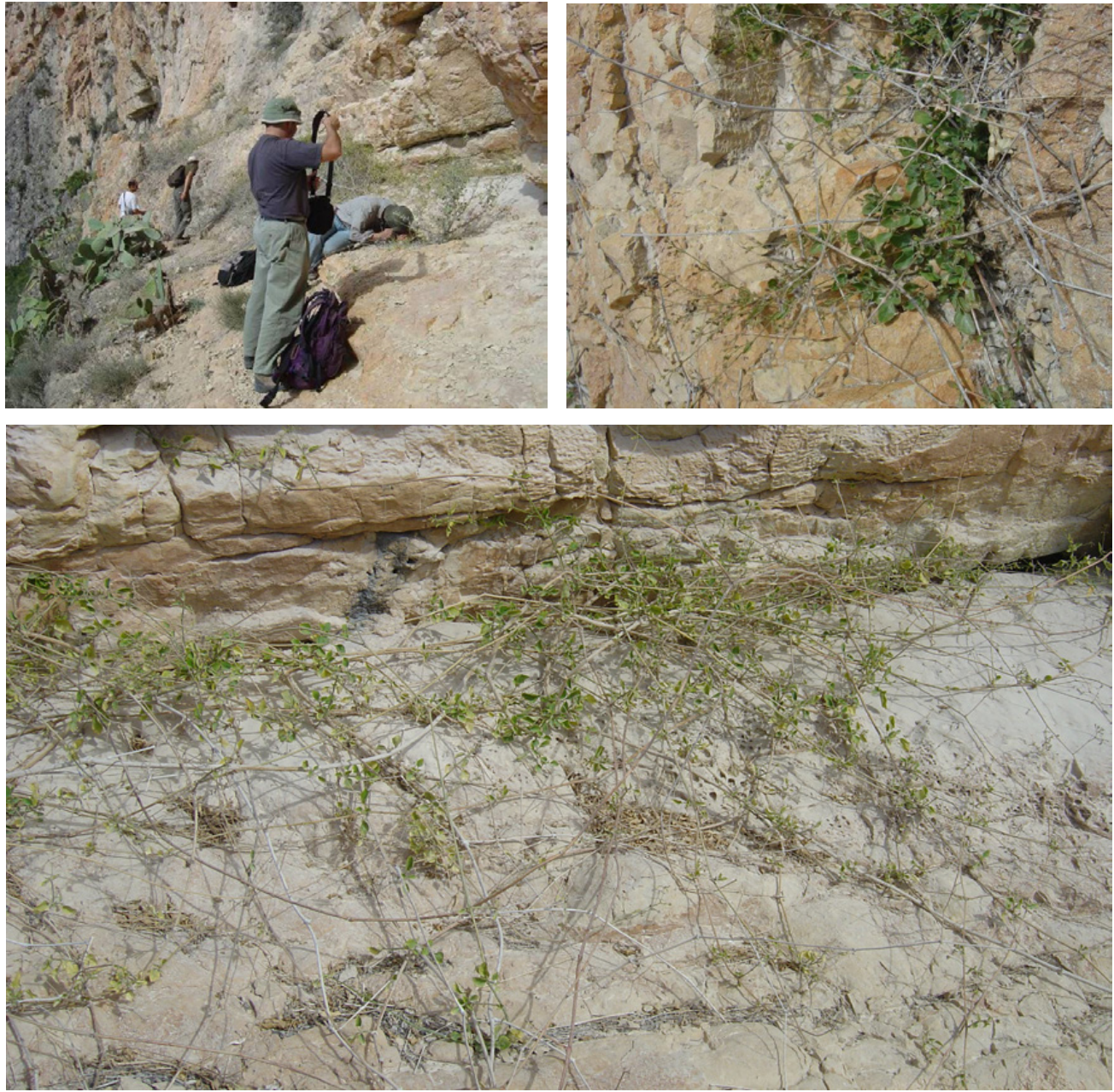

Figura 2. Población natural de Boerhavia repens en Teulada (Alicante), en abril de 2005. Desde 2012 no se han vuelto a encontrar ejemplares silvestres en esta zona. 

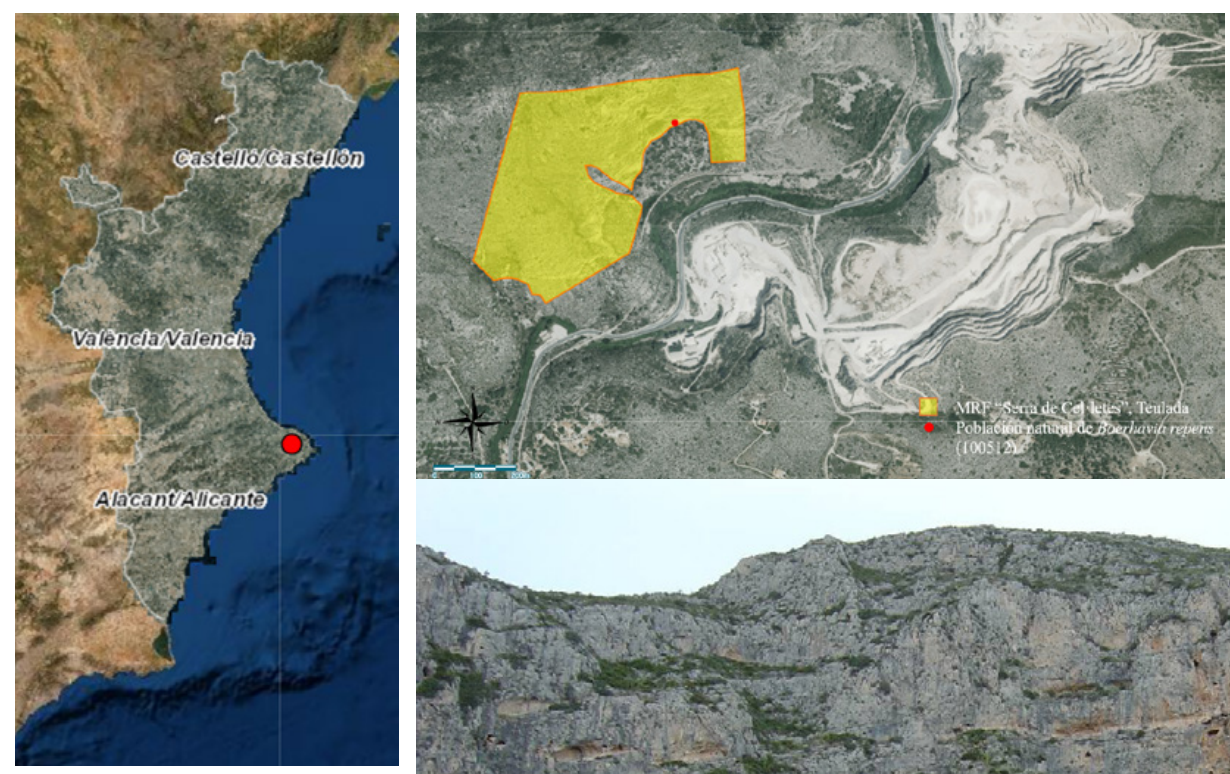

Figura 3. Localización de la población natural de Boerhavia repens dentro de la Microrreserva de flora "Serra de Cel-letes" (Teulada, Alicante) y panorámica del hábitat en 2012.
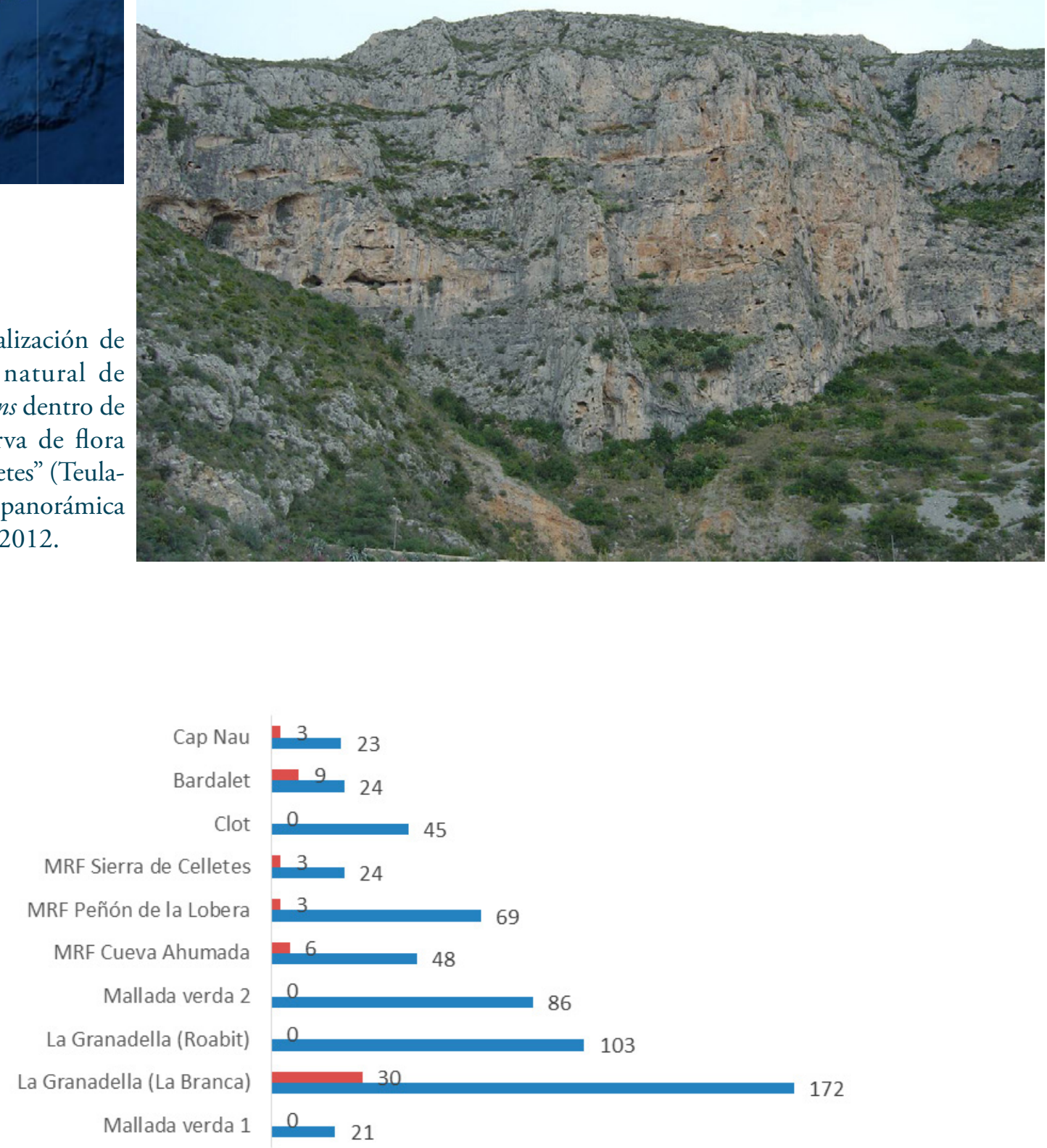

— № ejemplares último censo — № ejemplares restituidos

Figura 4. Número de ejemplares plantados en cada translocación de conservación realizadas para Boerhavia repens y la supervivencia observada en cada una de las acciones. 


\begin{tabular}{c|c|c|c|c|c|c|c} 
ID_UTS & $\begin{array}{c}\text { Unidad } \\
\text { translocada de } \\
\text { seguimiento }\end{array}$ & $\begin{array}{c}\text { Año inicio } \\
\text { de la } \\
\text { restitución }\end{array}$ & $\begin{array}{c}\text { Ejemplares } \\
\text { restituidos }\end{array}$ & $\begin{array}{c}\text { No } \\
\text { ejemplares } \\
\text { último } \\
\text { censo }\end{array}$ & $\begin{array}{c}\text { Año } \\
\text { último } \\
\text { censo }\end{array}$ & $\begin{array}{c}\text { Años } \\
\text { transcurridos } \\
\text { hasta último } \\
\text { censo }\end{array}$ & $\begin{array}{c}\text { Super- } \\
\text { vivencia } \\
\text { (\%) }\end{array}$ \\
\hline 455_03 & $\begin{array}{c}\text { Mallada verda 1, } \\
\text { Benissa }\end{array}$ & 2011 & 21 & 0 & 2012 & 1 & 0 \\
\hline 455_01 & $\begin{array}{c}\text { La Granadella (La } \\
\text { Branca), Xàbia }\end{array}$ & 2016 & 172 & 30 & 2017 & 1 & 17,4 \\
\hline 455_02 & $\begin{array}{c}\text { La Granadella } \\
\text { (Roabit), Xàbia }\end{array}$ & 2016 & 103 & 0 & 2019 & 3 & 0 \\
\hline 455_04 & $\begin{array}{c}\text { Mallada verda 2, } \\
\text { Benissa }\end{array}$ & 2016 & 86 & 0 & 2017 & 1 & 0 \\
\hline 455_05 & $\begin{array}{c}\text { MRF Cueva } \\
\text { Ahumada, Callosa } \\
\text { de Segura }\end{array}$ & 2018 & 48 & 6 & 2019 & 1 & 12,5 \\
\hline 455_06 & $\begin{array}{c}\text { MRF Peńón de la } \\
\text { Lobera, Redován }\end{array}$ & 2018 & 69 & 3 & 2019 & 1 & 4,3 \\
\hline 455_07 & $\begin{array}{c}\text { MRF Sierra de } \\
\text { Celletes, Teulada }\end{array}$ & 2018 & 24 & 3 & 2019 & 1 & 12,5 \\
\hline 455_09 & Clot, Elx & 2018 & 45 & 0 & 2019 & 1 & 0 \\
\hline B55_10 & Capdalet, Elx & 2018 & 24 & 9 & 2019 & 1 & 37,5 \\
\hline TOTAL & 2018 & 23 & 3 & 2019 & 1 & 13,0 \\
\hline
\end{tabular}

Tabla 1. Translocaciones de conservación realizadas con Boerhavia repens.

* UTS: Unidad translocada de seguimiento

Todas las experiencias han sido realizadas con plantones, para futuras experiencias se prevé la realización de sembrados, que hasta el momento no han podido realizarse por la falta de cantidad suficiente de semillas para acometer estos trabajos. En este sentido, toda la producción de planta que se ha realizado con esta especie parte de una recolección de 14 semillas realizada en la población natural (ver apartado 2.1 Recolección, caracterización y conservación de materiales de reproducción: frutos y semillas).

\section{Acciones de conservación ex situ}

\subsection{Recolección, caracterización y conservación} de materiales de reproducción: frutos y semillas. Evaluación de la viabilidad de las semillas recolectadas del suelo y de la planta.

En dos ocasiones se pudieron recolectar semillas de la población natural. La primera vez fue en 2005 y se recolectaron 14 semillas de 7 ejemplares, y en la segunda ocasión se recolectaron 20 semillas en el 


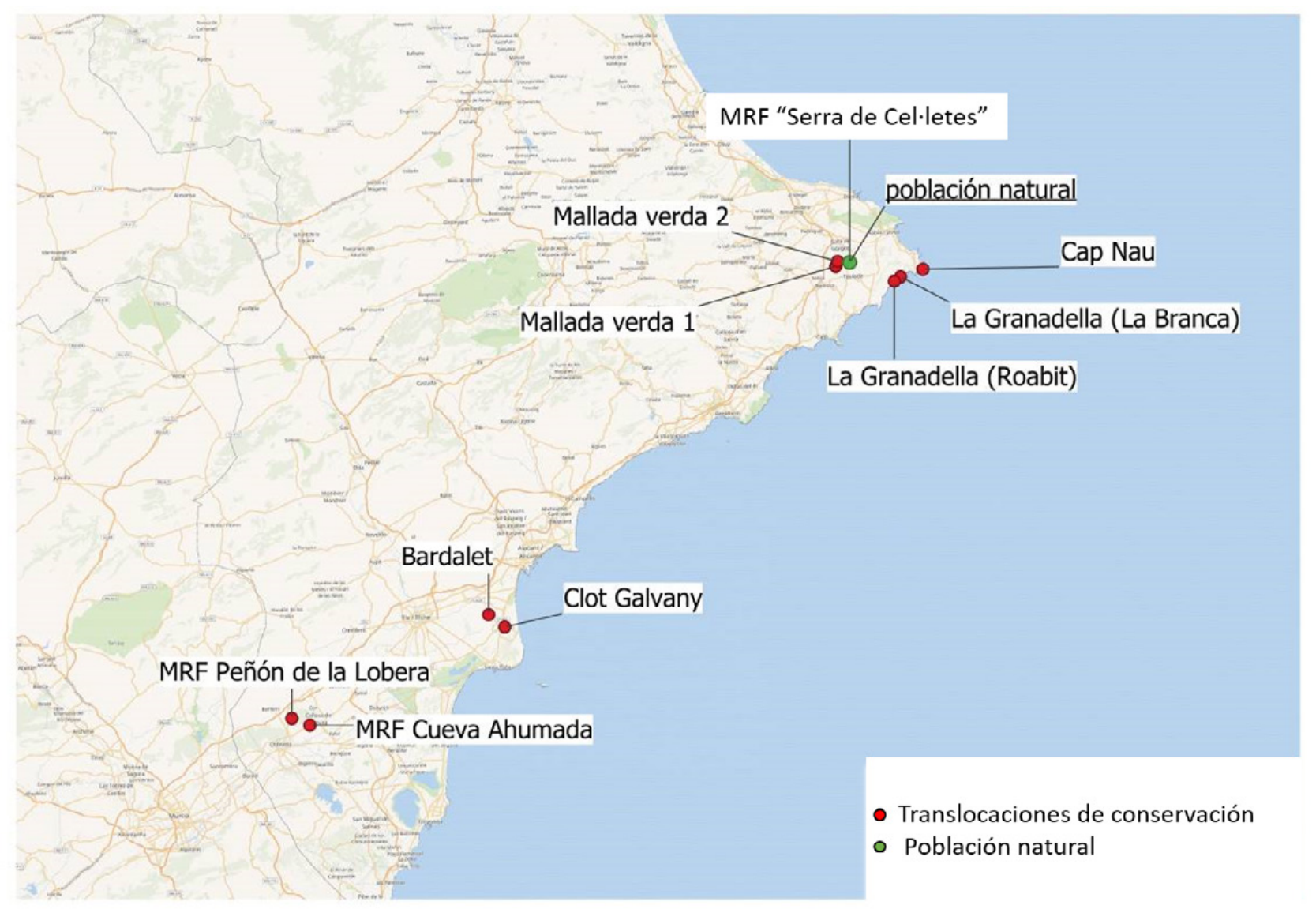

Figura 5. Localización de la población natural de Boerhavia repens y las translocaciones de conservación realizadas desde 2011 en la Comunidad Valenciana.

año 2007 (véase Ferrando-Pardo et al. 2008). Todo el material del primer lote se empleó para su germinación en ese mismo año, dando lugar a 7 plantas adultas reproductoras con las que se inició en 2006 una colección de plantas madre. A partir de este año, todas las anualidades se han podido recolectar semillas, lo que ha generado la conservación de 14 lotes con un total de 314.536 semillas aproximadamente (Figura 6). Estos lotes están disponibles en la colección activa del CIEF (Figura 3) y se han realizado dos duplicados para su conservación a largo plazo en el Banco de Germoplasma del Jardí Botànic de la Universitat de Valencia.

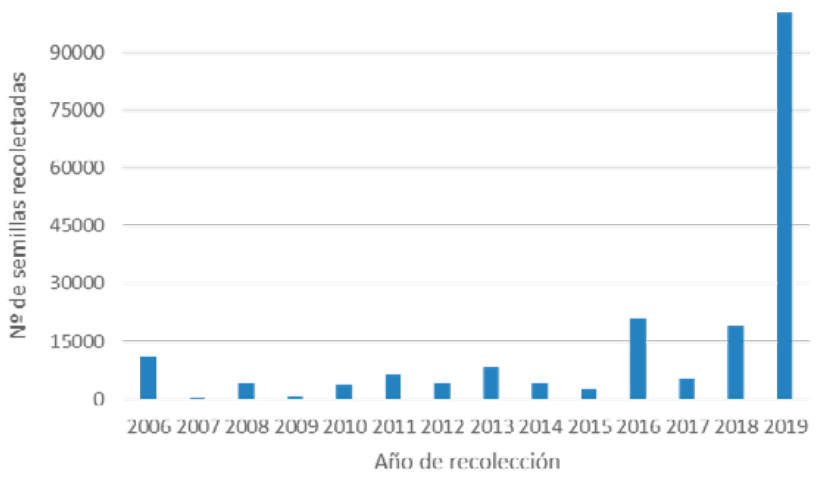

Figura 6. Cantidad de semillas de Boerhavia repens recolectadas por año procedentes de la colección de plantas mantenida en las instalaciones del CIEF. 
Las recolecciones de semillas se realizan durante los meses de junio y octubre. Este proceso es dificultoso debido a que los frutos son muy pegajosos, a causa de la existencia de glándulas que lo ornamentan en la superficie del epicarpio (una estructura que rodea al fruto), y además se recubren de mucílago cuando entran en contacto con el agua, lo que añade más dificultad a la recolección. Las semillas se recolectan manualmente de las plantas, pero una gran parte se cosechan una vez se han desprendido de la planta madre. En este punto, en lo que respecta a la recolección, y ante la sospecha de que las semillas pudieran perder viabilidad al desprenderse de las plantas y ser hidratadas con el riego, se ha realizado experimentación durante 2019 para contrastar algunas hipótesis.

Estas experiencias han consistido en el estudio de la viabilidad y la capacidad germinativa de las semillas mediante el test de tetrazolio y pruebas de germinación, utilizando los protocolos óptimos ya descritos para la especie. Para los análisis se utilizaron frutos y semillas recolectadas en el mismo año, diferenciando entre material recolectado directamente de la planta (julio, 2018) y del suelo (octubre, 2018). Se realizaron 4 réplicas de 25 semillas o frutos por cada test. Los resultados se muestran en la Tabla 2 y Figuras 7 y 8.

Existen diferencias en los porcentajes de germinación final según el momento y las condiciones de recolección de los frutos. El material que se recolectó directamente de la planta muestra germinaciones mayores (89\%) al material recolectado cuando los frutos han caído al suelo (25\%). Sin embargo, no se observan diferencias significativas en la capacidad germinativa entre ambos tipos de semillas, siendo en ambos casos muy próximos al 100\% (véase Tabla 2), ya que una gran parte de las semillas recolectadas del suelo que no germinaron durante el test de germinación resultaron estar frescas y viables $(71 \%)$ tras acometerse los análisis de caracterización al finalizar el test. Un 6\% de las semillas estaban muertas (Tabla 2, Figura 7).

Por otra parte, los test de viabilidad a través de la prueba de tetrazolio (Figura 8) confirman que hay un porcentaje de semillas del suelo que no están muertas, ni vacías, ni malformadas, por lo que se podría inferir que son viables y que podrían germinar si tuviesen las condiciones adecuadas, aunque el porcentaje de viabilidad observado $(62,5 \%)$ es inferior al encontrado en las semillas recolectadas directamente de la planta (93,7\%). En este sentido, parece que sí existe una pérdida de viabilidad $(37,5 \%)$ cuando los frutos caen al suelo y son recolectados días después desde su dispersión. Por otra parte, el hecho de que las semillas recolectadas del suelo no hayan germinado en el test de germinación podría deberse a la activación de mecanismos de letargo o dormiciones en las semillas.

El empleo de frutos en lugar de semillas en los test de germinación revela un retraso importante en el inicio de la germinación y en el porcentaje final de germinación transcurridos 45 días, pero no la

\begin{tabular}{|c|c|c|c|c|c|c|}
\hline \multicolumn{2}{|c|}{$\begin{array}{c}\text { Material de } \\
\text { reproducción utilizado }\end{array}$} & $\begin{array}{l}\text { Viabilidad (\%) } \\
(X \pm \text { S.D.) }\end{array}$ & $\begin{array}{l}\text { Germinación } \\
(\%)(X \pm \text { S.D. })\end{array}$ & $\begin{array}{l}\text { Capacidad } \\
\text { germinativa } \\
(\%)(X \pm \text { S.D. })\end{array}$ & $\begin{array}{l}\text { Semillas } \\
\text { muertas } \\
(\%)\end{array}$ & $\begin{array}{c}\text { Semillas } \\
\text { frescas } \\
(\%)\end{array}$ \\
\hline \multirow{2}{*}{$\begin{array}{l}\text { Recolección } \\
\text { de la planta }\end{array}$} & Semillas & $93,7 \pm 8,8$ & $89 \pm 6$ & $100 \pm 0$ & 0 & 11 \\
\hline & Frutos & - & $8 \pm 5,6$ & $96 \pm 4,6$ & 6 & 86 \\
\hline \multirow{2}{*}{$\begin{array}{l}\text { Recolección } \\
\text { del suelo }\end{array}$} & Semillas & $62,5 \pm 17,6$ & $25 \pm 8,87$ & $97 \pm 3,8$ & 4 & 71 \\
\hline & Frutos & - & $0 \pm 0$ & $90 \pm 6,9$ & 10 & 90 \\
\hline
\end{tabular}

Tabla 2. Resultados en los test de viabilidad (test de tetrazolio) y de germinación en semillas y frutos de Boerhavia repens recolectados en 2018 de la planta y del suelo. 


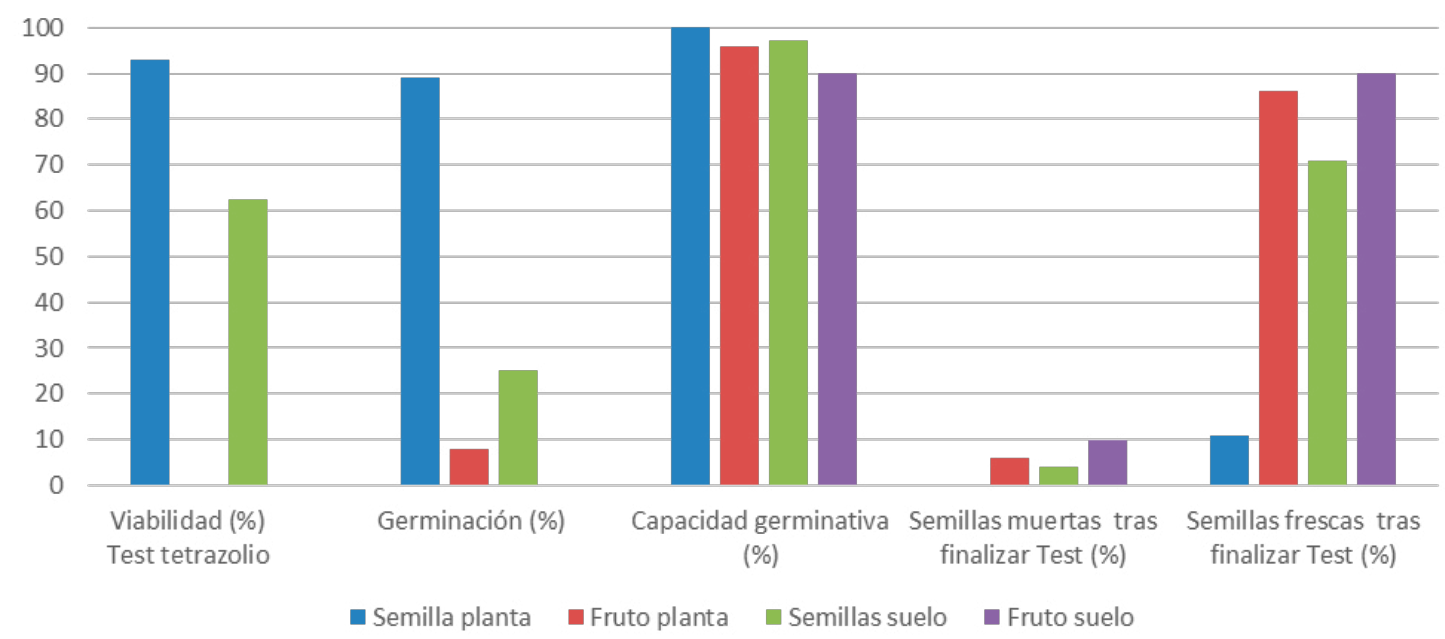

Figura 7. Resultados en los test de viabilidad (test de tetrazolio) y de germinación en semillas y frutos de Boerhavia repens recolectados en 2018 de la planta y del suelo.
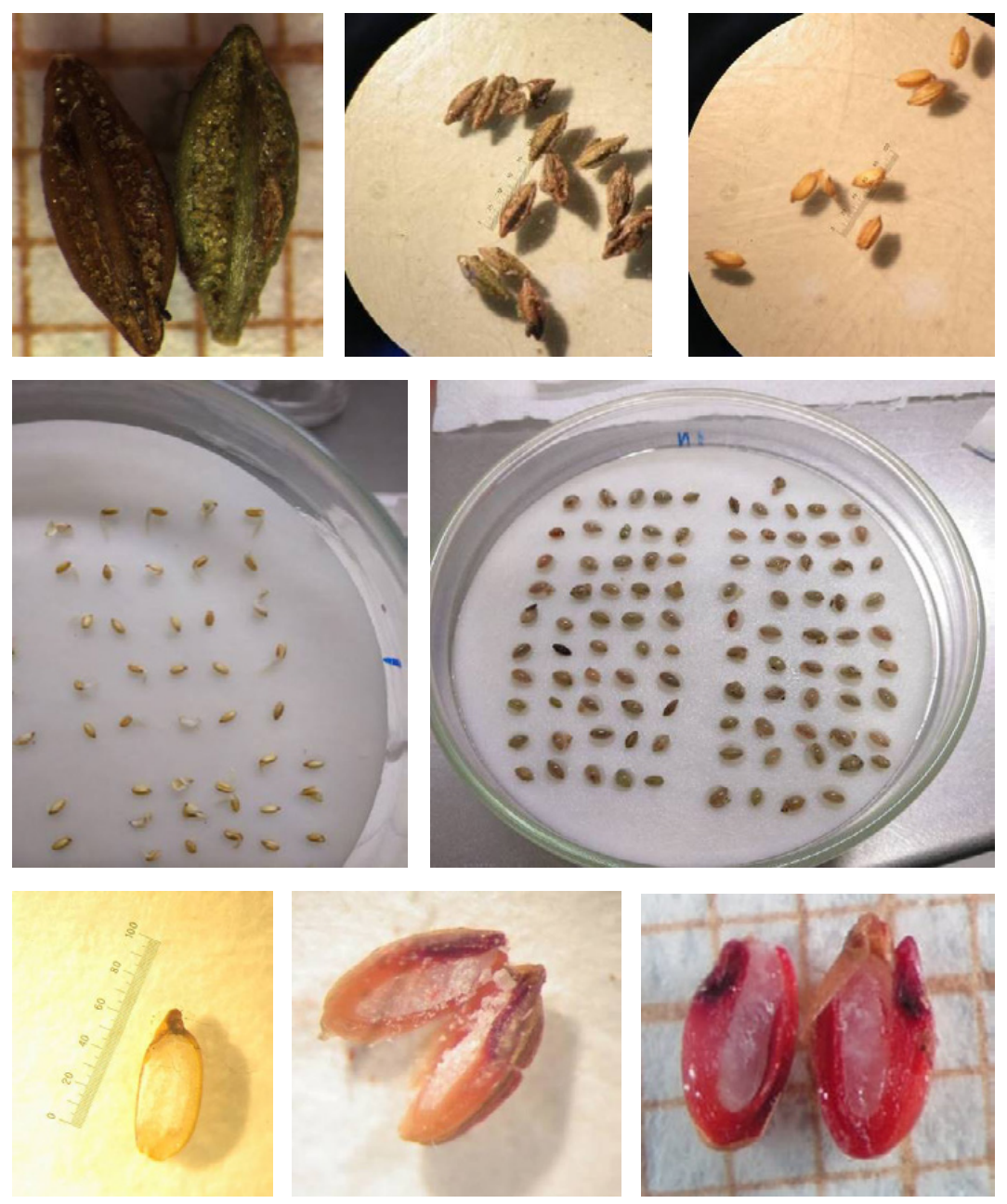

Figura 8. Arriba: caracterización de frutos (izquierda y centro) y semillas (derecha) de Boerhavia repens. Centro: test de germinación de semillas (izquierda) y de frutos (derecha). Abajo: criterios de evaluación para el test de viabilidad utilizando la tinción con tetrazolio, semilla no teñida, embrión no viable (izquierda), semilla con tinción parcial, embrión no viable (centro) y semilla viable totalmente teñida (derecha). 
imposibilita. Las semillas sin epicarpio germinan más rápido ( $89 \%$ en 8 días) que las que se encuentran en el interior de los epicarpios (20\% en 45 días). Tras la prueba de corte realizada a los frutos, una vez dado por finalizado el test de germinación, se observa que las semillas contenidas en ellos, en una gran proporción, son viables (86\%), otra observación más que documenta que las cubiertas son mecanismos de inhibición de la germinación, encontrándose un pequeño porcentaje de frutos con semillas muertas (6\%) y no encontrándose frutos vacíos.

\subsection{Producción de planta. Estudios de supervi- vencia de las plántulas}

La mejora del estado de conservación de esta especie requiere ineludiblemente de la propagación, multiplicación y producción de planta para realizar trabajos de translocaciones. Para la producción de planta se han utilizado tanto técnicas de multiplicación vegetativa, sobre todo en los primeros años en los que no había suficiente disponibilidad de semillas, como por reproducción sexual.

Desde 2006 se mantiene en el CIEF una colección de planta viva que cuenta con aproximadamente 65 ejemplares/año. En la figura 6 se muestra la cantidad de semillas que se han recolectado de este huerto a lo largo de los años durante los que se ha mantenido y gestionado.
Durante 2018 y 2019 se ha estudiado la supervivencia de las plántulas tras el repicado de las semillas germinadas en sustrato y en condiciones controladas de invernadero (Tabla 3). Se ha comparado los resultados de los repicados realizados en invierno, con semillas pre-germinadas durante los meses de noviembre y diciembre, y los realizados en primavera, entre marzo y abril. Se ha observado una mortalidad en las plántulas en las primeras fases del desarrollo independientemente de si las germinaciones ocurren en invierno o primavera, siendo la mortalidad de 66,9\% y $65,3 \%$, respectivamente (Tabla 3). Transcurrido esta primera etapa de desarrollo, cuando las germinaciones sucedieron en primavera, las plántulas que superaron esta fase, sobrevivieron en un $90 \%$ y consiguieron pasar a la fase adulta reproductora. Sin embargo, las plántulas que germinaron en noviembre sufrieron de una mortalidad del $82,3 \%$. En ambos casos las plantas que superaron la fase juvenil llegaron a adultas en el $100 \%$ de los casos. El 80\% de los ejemplares adultos reproductores rebrota al año siguiente al inicio de la primavera, durante el mes de marzo, el resto muere.

\section{CONCLUSIONES}

Desde 2012 no se han vuelto a encontrar ejemplares vivos silvestres en la población natural de Teulada. El último registro de esta población fue

\begin{tabular}{l|c|c|c|c|c|c|c|c|c}
\hline & \multicolumn{2}{|c|}{$\begin{array}{c}\text { Transcurrido 1 mes } \\
\text { (fase plántula) }\end{array}$} & \multicolumn{2}{|c|}{$\begin{array}{c}\text { Transcurridos 3 meses } \\
\text { (fase juvenil) }\end{array}$} & \multicolumn{2}{c}{$\begin{array}{c}\text { Transcurridos 6 meses } \\
\text { (fase adulto) }\end{array}$} \\
$\begin{array}{c}\text { de germi- } \\
\text { nación y } \\
\text { repicado }\end{array}$ & $\begin{array}{c}\text { No semillas } \\
\text { pre germi- } \\
\text { nadas repi- } \\
\text { cadas }\end{array}$ & $\begin{array}{c}\text { No plán- } \\
\text { tulas } \\
\text { desarro- } \\
\text { lladas }\end{array}$ & $\begin{array}{c}\text { Mortali- } \\
\text { dad (\%) }\end{array}$ & $\begin{array}{c}\text { No plán- } \\
\text { tulas } \\
\text { desarro- } \\
\text { lladas }\end{array}$ & $\begin{array}{c}\text { Mortali- } \\
\text { dad (\%) }\end{array}$ & $\begin{array}{c}\text { Supervi- } \\
\text { vencia res- } \\
\text { pecto a fase } \\
\text { plántula } \\
\text { (\%) }\end{array}$ & $\begin{array}{c}\text { No plan- } \\
\text { tas desa- } \\
\text { rrolladas }\end{array}$ & $\begin{array}{c}\text { Mortali- } \\
\text { dad (\%) }\end{array}$ & $\begin{array}{c}\text { Super- } \\
\text { vivencia } \\
\text { respecto a } \\
\text { fase juvenil } \\
\text { (\%) }\end{array}$ \\
\hline Primavera & 346 & 120 & 65,3 & 108 & 68,7 & 90,0 & 108 & 0 & 100 \\
\hline Invierno & 130 & 43 & 66,9 & 23 & 82,3 & 53,4 & 23 & 0 & 100
\end{tabular}

Tabla 3. Resultados en la supervivencia de las semillas pre-germinadas transcurridos 1, 3 y 6 meses tras su repicado en contenedores de cultivo. 
de 4 individuos (2012) no ocupando más de $2 \mathrm{~m}^{2}$. En este sentido, los factores y amenazas que podrían haber marcado el rápido declive y finalmente la pérdida de la única población europea de $B$. repens podrían haber sido el escaso número de individuos de la población, el envejecimiento de las plantas y ausencia de reclutamiento, la actividad minera cercana y los periodos de sequía prolongados.

Se ha observado una alta mortalidad en las plántulas en las primeras fases del desarrollo en ejemplares en cultivo (entre un 65 y 67\%). Transcurrido esta primera etapa, si las germinaciones suceden en primavera, el $90 \%$ de las plántulas pasan a una fase juvenil y el $100 \%$ de los juveniles a adultos reproductores. El 80\% de los ejemplares adultos reproductores rebrota al ańo siguiente al inicio de la primavera (marzo), el resto muere. Si las germinaciones suceden en invierno un alto porcentaje de plántulas y de juveniles mueren y pocos llegan alcanzar la etapa de adulto reproductor.

Desde 2006 se mantiene en el CIEF una colección de planta viva que cuenta con aproximadamente 65 ejemplares/año. Se conservan 15 lotes de semillas (1 recolectado de la población natural y el resto procedentes de la colección de planta viva) con un total de 314.536 semillas aproximadamente. Las semillas pierden viabilidad $(37,5 \%)$ si los frutos no son recolectados directamente de la planta. Las cubiertas de los frutos (epicarpio) retardan la germinación y en un porcentaje alto (próximos al 90\%) la inhiben. Esto podría deberse a la activación de mecanismos de letargo y dormiciones morfológicas en las semillas al dispersarse los frutos.

Desde 2011 se han realizado 10 plantaciones mediante translocaciones de conservación para las que se han utilizado un total de 615 plantas. Éstas han consistido en un refuerzo poblacional (MRF "Serra de Cel.letes") y 9 introducciones. El porcentaje de supervivencia medio de los ejemplares observados en las 10 translocaciones es de 11,8 $\pm 11,9$ trascurridos entre 1 y 3 años desde la plantación. Consideramos que ha transcurrido poco tiempo para poder evaluar el éxito de estos trabajos, pero sin duda es necesario un incremento de las plantaciones en un mayor número de sitios y con un mayor número de plantas, además de realizar experiencias de sembrado.

Estos datos son de especial interés, ya que a pesar del óptimo africano de distribución de la especie, ésta no exhibe el patrón fenológico habitual de los arbustos íbero-norteafricanos, que son caducifolioestivales (por ejemplo, Withania frutescens (L.) Pauquy, Lycium intricatum Boiss., Asparagus albus L.), y germinan y emiten sus hojas en el otońo. Al germinar y rebrotar preferentemente en primavera, $B$. repens demuestra una mayor termicidad -el reclutamiento es más efectivo en la época de aumento de la temperatura- y una mayor dependencia de las precipitaciones primaverales, lo que a su vez ayuda a explicar que la planta haya podido sobrevivir en el $\mathrm{N}$ de Alicante. Por el contrario, hacia el S de la provincia, aunque los inviernos sean menos crudos, la mayor escasez de precipitaciones primaverales actuaría en contra de la instalación de la especie. Estos aspectos podrán comprobarse mejor en el futuro, a partir de la supervivencia y presencia de posibles nuevas generaciones. Boerhavia repens es una de las especies cuyas poblaciones se habría extinguido en época reciente, si bien lo indicado sobre la pervivencia de las semillas y su capacidad reproductiva aconseja seguir revisando periódicamente la población de Teulada por si se sucedieran germinaciones. La diversidad de hábitats, y en especial la dualidad entre su comportamiento ibérico y norteafricano, permite recomendar que se desarrollen experiencias adicionales de translocaciones de conservación en hábitats más favorables, como podrían ser los de tipo dunar o acantilados terrosos costeros con orientación favorable.

Por último, consideramos interesante resaltar la dificultad que supone trabajar en la recuperación de una especie para la que se tiene muy poca información sobre su ecología en el territorio donde se desarrollan las actividades de conservación. En este sentido, la falta de referencias sobre los hábitats donde la especie puede estar supone un hándicap en el éxito de las actuaciones de translocación. Sin duda la presencia de $B$. repens en la flora europea en una exigua población alicantina, es un claro ejemplo de ello. En este sentido, creemos que realizar experien- 
cias controladas para conocer la idoneidad del tipo de hábitat para esta especie en la Comunidad Valenciana ayudará sin duda el éxito de las translocaciones como herramienta fundamental para la recuperación de esta especie en el territorio valenciano.

\section{AGRADECIMIENTOS}

Los autores agradecen a Mónica Lloret y Nerina Pérez la ayuda prestada en el estudio de la viabilidad de las semillas, así como de la supervivencia de las plantas en cultivo. Agradecemos también a los compañeros Víctor Martínez, Bernat Plana e integrantes de las Brigadas Natura 2000 de Alicante el trabajo realizado dentro de este proyecto de conservación.

\section{REFERENCIAS}

AFRICAN PLANT DATABASE (2012). Version 3.3. Conservatoire et Jardin botaniques de la Ville de Genève and South African National Biodiversity Institute, Pretoria. http://www.ville-ge.ch/musinfo/bd/cjb/africa/ details.php?langue $=$ an $\&$ id $=149125$

Aguilella, A., Fos, S. \& Laguna, E. (eds.), (2010). Catálogo Valenciano de Especies de Flora Amenazadas. Colección Biodiversidad, 18. Conselleria de Medi Ambient, Aigua, Urbanisme i Habitatge, Generalitat Valenciana, Valencia, 358 pp.

Anónimo (2009). Decreto 70/2009, de 22 de mayo, del Consell, por el que se crea y regula el Catálogo Valenciano de Especies de Flora Amenazadas y se regulan medidas adicionales de conservación. Diari Oficial de la Comunitat Valenciana, 6021: 20143-20162.

Anónimo (2013). Orden 6/2013, de 25 de marzo, de la Consellería de Infraestructuras, Territorio y Medio Ambiente, por la que se modifican los listados valencianos de especies protegidas de flora y fauna. Diari Oficial de la Comunitat Valenciana, 6996: 8682-8690.

Bolòs, O., Vigo, J., Masalles, R.M., \& Ninot, J.M. (2005). Flora manual dels Països Catalans. 3a Edició, Ed. Pòrtic, Barcelona.
Chen, S.-H. \& WU, M.-J. (2007). A taxonomical study of the genus Boerhavia (Nyctaginaceae) in Taiwan. Taiwania, 52: 332-342.

Codd, L.E. (1966). Notes on Boerhavia in southern Africa. Bothalia, 9: 113-121.

Crespo, M.B. (2004). Boerhavia repens L. subsp. repens. In: Bañares, A. \& al. (eds.), Atlas y libro rojo de la flora vascular amenazada de España: 144-145. Organismo Autónomo Parques Nacionales., Ministerio del Medio Ambiente. Madrid.

De Albuquerque, U.P., De Medeiros, P.M., De Almeida, A.L.S., Monteiro, J.M., De Freitas Lins Neto, E.M., De Melo, J.G. \& Dos Santos, J.P. (2007). Medicinal plants of the caatinga (semi-arid) vegetation of NE Brazil: a quantitative approach. Journal of Ethnopharmacology, $114: 325-354$

Ferrando-Pardo, I., Ferrer-Gallego, P.P., Navarro, A. \& Laguna, E. (2008). Acciones de conservación ex situ de la población europea de Boerhavia repens L. subsp. repens (Nyctaginaceae). Flora Montiberica, 39:19-32.

Ferrando-Pardo, I. Ferrer-Gallego, P.P. \& LagunaLumbreras, E. (2016) Assessing the conservation value of ex situ seed bank collections of endangered wild plants. Israel Journal of Plant Sciences, 63(4):333-346, DOI: $10.1080 / 07929978.2015 .1125676$

Ferrer-Gallego, P.P., Ferrando-Pardo, I., Gago, C. \& Laguna, E. (eds.), (2013). Manual para la conservación de germoplasma y el cultivo de la flora valenciana amenazada. Colección Manuales Técnicos Biodiversidad, 3. Conselleria d'Infraestructures, Territori i Medi Ambient, Generalitat Valenciana, Valencia, 246 pp. http://www. agroambient.gva.es/es/web/biodiversidad/manual-parala-conservacion-de-germoplasma-y-el-cultivo-de-laflora-valenciana-amenazada

Klopper, R.R., Chatelain, C., Bänninger, V., Habashi, C., Steyn, H.M., De Wet, B.C., Arnold, T.H., Gautier, L., Smith, G.F. \& Spichiger, R. (2006). Checklist of the flowering plants of Sub-Saharan Africa. An index of accepted names and synonyms. South African Botanical Diversity Network Report No 42. SABONET, Pretoria.

Laguna, E. \& Ferrer-Gallego, P.P. (2012). Reforzamientos de proximidad y Neopoblaciones de seguridad, nuevos conceptos complementarios para determinados tipos de implantaciones vegetales in situ. Conservación Vegetal, 16: 14 . 
Lehman, A.D., Dunkel, F.V., Klein, R.A., Ouattara, S., Diallo, D., Gamby, K.T. \& N'Diaye, M. (2007). Insect management products from Malian traditional medicine - establishing systematic criteria for their identification. Journal of Ethnopharmacology, 110: 235249.

Maire, R. (1962). Flore de l'Afrique de Nord, 8, Paris. Rue de Tournon.

Mateo, G. \& Crespo, M.B. (2014). Claves ilustradas para la flora valenciana. Ed. Jolube. Jaca.

Muzila, M. (2006). Boerhavia diffusa L. In: Schmelzer, G.H. \& Gurib-Fakim, A. (Eds.), PROTA (Plant Resources of Tropical Africa/Ressources végétales de l'Afrique tropicale), Wageningen.
Saikia, A.P., Ryakala, V.K., Sharma, P., Goswami, P. \& Bora, U. (2006). Ethnobotany of medicinal plants used by Assamese people for various skin ailments and cosmetics. Journal of Ethnopharmacology, 106: 149-157.

Soler, J.X. (1995). Primera cita de Boerhavia repens L. (Nyctaginaceae) para la flora ibérica. Anales del Jardin Botánico de Madrid, 53: 123-125.

Struwig, M. \& Siebert, S.J. (2013). A taxonomic revision of Boerhavia (Nyctaginaceae) in southern Africa. South African Journal of Botany, 86: 116-134. 